\title{
Aplicación de una Metodología Estructurada para el Diseño de un Sistema de Cosecha Selectiva de Café
}

\author{
Aplicación de una Metodología Estructurada para el Diseño de un Sistema de Cosecha \\ Selectiva de Café
}

\author{
Paula Jimena Ramos Giraldo, Mauricio García Navarro, Juan Felipe Hoyos Suárez, Carlos Eugenio Oliveros \\ Tascón, Juan Rodrigo Sanz Uribe \\ Centro Nacional de Investigaciones en Café - Federación Nacional de Cafeteros de Colombia, Chinchiná, Colombia \\ paula.ramos@cafedecolombia.com \\ maurogn1@gmail.com \\ juanfehs20@gmail.com \\ carlos.oliverosecafedecolombia.com \\ juanr.sanz@cafedecolombia.com
}

\begin{abstract}
Resumen-Se usó una metodología para el diseño de dispositivos que permitan el desprendimiento selectivo de frutos de café. Se tuvieron en cuenta especificaciones técnicas del producto deseado y las condiciones generadas por los frutos de café en ramas. Se generaron 33 opciones viables técnicamente de las cuales fueron seleccionadas las mejores tres calificadas para ser exploradas y finalmente seleccionar solo una para ser diseñada de manera detallada. Dicha opción fue evaluada en condiciones de laboratorio y mostró un porcentaje de desprendimiento de frutos maduros del $60 \%$ y de frutos verdes del $20 \%$.
\end{abstract}

Palabras clave- Metodología estructurada de diseño, cosecha selectiva, café, micromecanismos.

Abstract-A design methodology was used to obtain devices for selective detachment of coffee fruits. The technical specifications of the desire product were taken into account, as well as the conditions offered by the coffee fruits in the branches. There were generated 33 viable options of which the three best qualified were selected for being explored. Then it was chosen the most viable option to be designed in detail. The final device was evaluated in lab conditions and the detached mass showed a mature fruits content of $60 \%$ and an immature fruits content of $20 \%$.

Key Words - Structured design methodology, harvest, coffee, micromechanisms.

\section{INTRODUCCIÓN}

La recolección manual de café es la actividad con mayor participación en los costos de producción de café en Colombia. Para disminuir estos costos se han evaluado equipos utilizados en otros países en la cosecha de café y de otros productos agrícolas, y de diseño propio, con los cuales se han observado importantes avances en el rendimiento de la mano de obra, aunque sin obtener la calidad y eficacia de desprendimiento de frutos maduros esperada en Colombia. El alto número de floraciones en el año, que dan lugar a múltiples pases de recolección (hasta 20 en el año), con baja carga y concentración de frutos maduros en la mayoría de ellos dificulta el empleo exitoso de equipos para la cosecha de café en Colombia, portátiles principalmente. Para lograr mejores resultados en la cosecha del café en las condiciones de la caficultura colombiana, se utilizó una metodología estructurada de diseño, la cual permite considerar la complejidad de un problema de diseño mecánico en forma ordenada y sistémica para plantear alternativas tecnológicas que permitan alcanzar los resultados esperados.

\section{ANTECEDENTES}

Los diferentes trabajos desarrollados hasta el momento para la recolección de café en la disciplina de ingeniería agrícola de Cenicafé han contribuido a mejorar considerablemente los indicadores de la recolección, principalmente la eficiencia, utilizando herramientas portátiles que permiten actuar directamente sobre los glomérulos, en particular el DESCAFE [10], equipo que realiza el proceso de desprendimiento de frutos alrededor de la rama, o versiones de los equipos ALFA [9] e IMFRA [7] los cuales permiten realizar el desprendimiento por caras sobre la rama. Estos equipos han mostrado resultados con eficacias mayores a las obtenidas mediante la cosecha manual tradicional, cuando las condiciones de maduración de los árboles, carga y concentración, son relativamente altas. Adicionalmente, se han desarrollado equipos que permiten asistir la cosecha manual, como es el caso del el equipo Canguaro $2 \mathrm{M}$ y su versión previa, como el equipo AROANDES [5], en los cuales se combinan los desprendimientos selectivos de frutos individuales de la cosecha manual tradicional junto con elementos de recibo de frutos. 


\section{METODOLOGÍA ESTRUCTURADA DE DISEÑO}

La Metodología Estructura de Diseño (DEM), [2], [3], [12], es una estrategia aplicada a diseño de productos, mediante la cual se permite abordar de manera ordenada un problema y generar alternativas objetivas para la concepción de una solución, desde diferentes aspectos como, el técnico y económico, entre otros.

La estrategia utilizada en la metodología estructurada de diseño se muestra en la Figura 1 y sigue los siguientes pasos para la herramienta de cosecha:

a. Identificación del problema, donde se formula claramente el problema a resolver con un producto final.

b. Formulación de especificaciones de diseño y su importancia en la solución.

c. Generación de diferentes alternativas para cada función que cumple la solución y disposición de estas en un espacio denominado Carta Morfológica, con la cual se generan opciones de diseño al combinar diferentes alternativas para cada función.

d. Evaluación de las opciones a partir del peso de las especificaciones de diseño y la matriz de PUGH [8].

e. Selección de la opción mejor calificada.

f. Diseño detallado y análisis de la opción seleccionada.

g. Chequeo del diseño, en caso de cumplir con los requisitos planteados se procede a la construcción, en caso contrario se debe seleccionar una opción diferente (paso f).

h. Pruebas exploratorias, en caso de cumplir con los objetivos, se procede a la evaluación final, en caso contrario se debe modificar la opción construida o seleccionar nuevamente otra opción.

i. Reporte de los resultados finales.

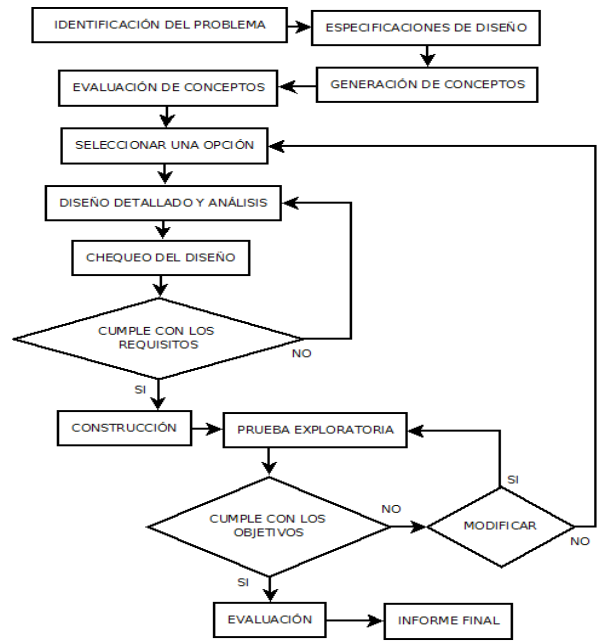

Figura 1. Diagrama de flujo metodología estructurada de diseño

\section{A. Identificación del problema.}

No existe un sistema de cosecha semimecanizada de café que bajo diferentes ofertas de frutos maduros en los árboles logre ser selectivo, rápido y económico. El producto que se ha propuesto como solución a este problema es la herramienta que coseche de forma individual y selectiva cada fruto de café, conformado por un sistema de desprendimiento por activación automática.

\section{B. Especificaciones del diseño.}

Para dos de los sistemas que conforman la herramienta, fueron seleccionados cinco aspectos principales y su ponderación en importancia, Tabla 1. Cada aspecto a su vez cuenta con diversas características que permiten analizar el diseño de las funciones a cumplir por la herramienta, Tabla 2.

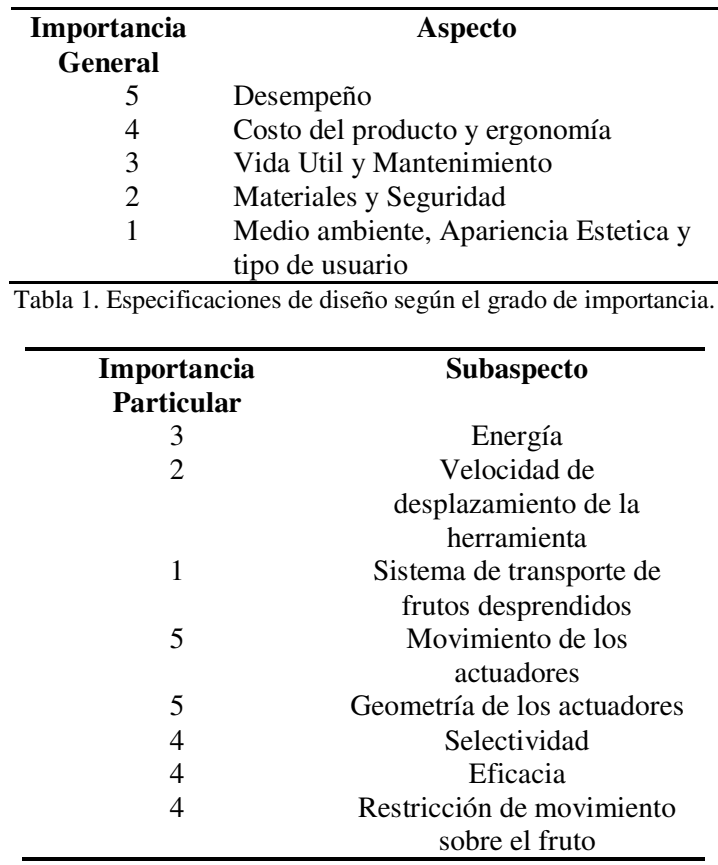

Tabla 2. Características particulares para el aspecto desempeño.

\section{Generación de conceptos}

Sobre una lista de funciones que debe cumplir la herramienta, se generaron diferentes alternativas para cada función a través de un enfoque de grupo, por medio de una lluvia de ideas y de entrevistas individuales, la lista de funciones se puede ver en la primera columna de la Tabla 3 y las alternativas en las subsiguientes.

Se obtuvieron para el sistema de desprendimiento 108 opciones, de las cuales solo 33 fueron consideradas viables técnicamente. En la Tabla 4 se pueden observar tres de estas opciones. 


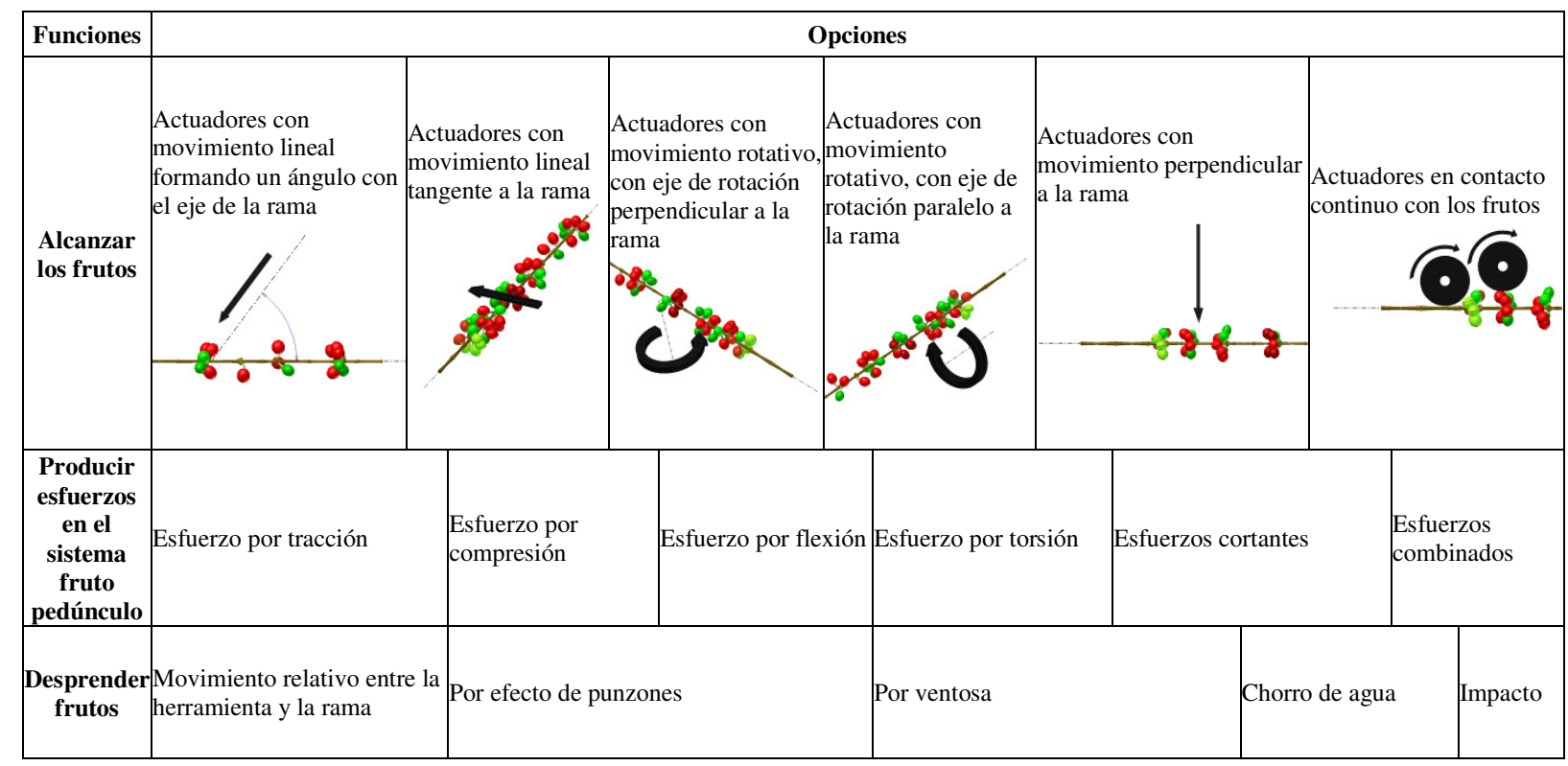

Tabla 3. Carta morfológica.

\begin{tabular}{|c|c|c|}
\hline \multicolumn{3}{|c|}{ Sistema de desprendimiento } \\
\hline Alcanzar los frutos & Producir esfuerzos en el sistema fruto pedúnculo & Desprender frutos \\
\hline Movimiento radial a la rama & Salida del actuador & Activación de una ventosa \\
\hline Movimiento axial a la rama & Por movimiento relativo entre la herramienta y la rama & $\begin{array}{l}\text { Gancho superficie punzones, } \\
\text { superficie alto agarre }\end{array}$ \\
\hline $\begin{array}{l}\text { Movimiento angular y movimier } \\
\text { la rama }\end{array}$ & $\begin{array}{l}\text { Por movimiento relativo entre la herramienta y la } \\
\text { rama, fricción entre los actuadores y los frutos, por } \\
\text { frenado de las esferas cuando se identifica un fruto } \\
\text { maduro }\end{array}$ & $\begin{array}{l}\text { Esferas de caucho, superficie } \\
\text { con alto agarre }\end{array}$ \\
\hline
\end{tabular}

Tabla 4. Algunas opciones generadas en la Carta Morfológica 


\section{Evaluación de conceptos}

Cada sistema fue tratado independientemente para realizar la evaluación de las especificaciones y subaspectos a partir de la matriz de PUGH. Se seleccionó una opción patrón, con la cual se compararon las demás opciones; finalmente se seleccionaron tres opciones para evaluar el funcionamiento técnico de las mismas y obtener criterios de evaluación para la selección final de la opción más viable.

En la Tabla 5, se muestra el resultado alcanzado por cada opción en cada especificación de diseño luego de comparar los subaspectos de cada opción con los subaspectos de la opción patrón, obteniendo calificaciones de "0" para subaspectos similares " +1 " para superiores y "-1" para inferiores, se obtiene el valor de cada especificación a partir de una suma ponderada por importancia de cada uno de los subaspectos y a su vez la calificación final es una suma ponderada de cada una de las especificaciones de diseño.

\begin{tabular}{|c|c|c|c|c|c|c|c|c|c|c|c|c|c|c|c|c|c|}
\hline & Op1 & Op2 & Op5 & Op6 & Op7 & Op10 & Op11 & Op12 & Op13 & Op14 & Op17 & Op18 & Op19 & Op20 & Op23 & Op24 & Op25 \\
\hline Desempeño & -7 & -5 & -1 & -3 & -1 & 3 & -3 & -3 & 9 & 3 & 1 & 1 & 0 & 2 & 0 & 0 & -2 \\
\hline Ergonomía & -3 & -3 & -3 & -3 & -3 & -3 & -3 & -3 & -3 & -3 & -3 & -3 & 0 & 0 & 0 & 0 & -3 \\
\hline Materiales & 0 & 0 & 0 & 0 & 0 & 0 & 2 & 2 & 0 & 0 & 2 & 2 & 0 & 0 & 2 & 2 & 0 \\
\hline Mantenimiento & -3 & -1 & -2 & -1 & -1 & -2 & -2 & -2 & -1 & -1 & & & & & & & \\
\hline Costo del producto & 8 & 8 & 8 & 8 & 8 & -8 & -8 & -8 & 8 & 8 & -8 & -8 & 0 & 0 & -8 & -8 & -8 \\
\hline Medio Ambiente & 0 & 0 & 0 & 0 & 0 & 0 & 0 & 0 & 0 & 0 & 0 & 0 & 0 & 0 & 0 & 0 & 0 \\
\hline Apariencia estetica & 0 & 0 & 0 & 0 & 0 & 0 & 0 & 0 & 0 & 0 & 0 & 0 & 0 & 0 & 0 & 0 & 0 \\
\hline Seguridad & 0 & 0 & 0 & 0 & 0 & 0 & 0 & 0 & 0 & 0 & 0 & 0 & 0 & 0 & 0 & 0 & 0 \\
\hline Total & -24 & -8 & 9 & 2 & 12 & -35 & -61 & -61 & 62 & 32 & -35 & -35 & 0 & 10 & -28 & -28 & -54 \\
\hline
\end{tabular}

Tabla 5. Evaluación de opciones matriz PUGH para el sistema de detección.

\section{Disposición del sistema de desprendimiento.}

Para encontrar la disposición de los sistemas de detección y desprendimiento, se realizó un análisis de las características típicas de una rama de café, sus glomérulos y sus frutos, tomando la información registrada para café Variedad Colombia [1], [4], [11]. En la Tabla 6 se muestran características máximas encontradas, sin embargo, estas pueden cambiar en menor o mayor proporción dependiendo de las condiciones climáticas del cultivo, las progenies que conforman la variedad, los sistemas de cultivo y ataque por plagas o enfermedades. En la Tabla 7 se muestran las características mínimas de la herramienta a desarrollar conforme a lo mostrado en la Tabla 6.

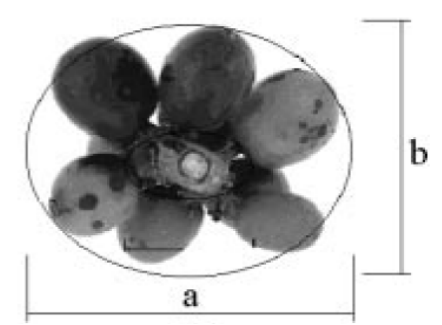

Figura 2. Frutos vistos de forma transversal en una rama. Las variables a y b corresponde a las elipses medidas en los nudos [4].

\begin{tabular}{|c|c|c|}
\hline $\begin{array}{c}\text { Características de la } \\
\text { herramienta }\end{array}$ & Detalle & Justificación \\
\hline Forma & $\begin{array}{l}\text { Cilíndrica o } \\
\text { semicilíndrica }\end{array}$ & $\begin{array}{l}\text { Tomar } 360^{\circ} \text { un } \\
\text { glomérulo }\end{array}$ \\
\hline $\begin{array}{l}\text { Tamaño de elipse que } \\
\text { alberga la herramienta }\end{array}$ & $66,21 \mathrm{~cm}$ & $\begin{array}{l}\text { Correspondiente a Eje } \\
\text { mayor elipse externa } \\
\text { (a) }[\mathrm{mm}] \text { Figura } 2\end{array}$ \\
\hline $\begin{array}{l}\text { Distancia adicional para } \\
\text { disponer sistema opto- }\end{array}$ & $40 \mathrm{~mm}$ & $\begin{array}{l}\text { Magnitud inicial } \\
\text { puede modificarse }\end{array}$ \\
\hline
\end{tabular}




$\begin{array}{lcc}\text { electrónico } & 107 \mathrm{~mm} & \text { Alberga los } 66,21 \mathrm{~cm} \\ \begin{array}{l}\text { Sección cilíndrica con un } \\ \text { diámetro }\end{array} & 6 & \begin{array}{c}\text { démero máximo de } \\ \text { frutos por corte } \\ \text { transversal }\end{array} \\ \begin{array}{l}\text { Puntos de visión } \\ \text { equidistantes }\end{array} & & \text {. }\end{array}$

Tabla 7. Especificaciones de diseño para tamaño y forma de la herramienta.

\section{RESULTADOS}

Luego de tener 33 opciones viables técnicamente como resultado DEM, fueron priorizadas 3 opciones de diseño para ser evaluadas en el desprendimiento individual de frutos de café. Cada opción buscaba a través de la combinación de materiales, geometría, y funcionalidad, cumplir principalmente con tres funciones: alcanzar los frutos, producir esfuerzos en el sistema fruto pedúnculo y desprender los frutos de café. Fueron considerados para alcanzar los frutos diferentes tipos de movimientos en los actuadores alrededor de la rama: actuadores con movimiento lineal, con desplazamiento axial, movimiento giratorio, movimiento angular, movimiento radial, y actuadores en contacto directo y continuo con los frutos. Con relación a los esfuerzos generados en el sistema fruto pedúnculo, fueron considerados: Esfuerzos por tracción, por compresión, por flexión, por torsión, esfuerzos cortantes y esfuerzos combinados. El sistema de desprendimiento es concebido como un arreglo circular de actuadores con el fin de lograr una cobertura de 360 grados alrededor del eje de la rama. En la Tabla 8 se muestran las características de las tres opciones a evaluar.

\begin{tabular}{|c|l|l|l|}
\hline Opción & \multicolumn{1}{|c|}{ Alcanzar los frutos } & \multicolumn{1}{c|}{$\begin{array}{c}\text { Producir esfuerzos en el sistema fruto } \\
\text { pedúnculo }\end{array}$} & Desprender frutos \\
\hline Torsión - Op 64 & Movimiento radial hacia la rama. & $\begin{array}{l}\text { Por movimiento relativo entre la herramienta y la } \\
\text { rama, fricción entre los actuadores y los frutos o } \\
\text { por frenado los actuadores en presencia de los } \\
\text { frutos con madurez de cosecha }\end{array}$ & $\begin{array}{l}\text { Cono invertido - superficie de alto } \\
\text { agarre }\end{array}$ \\
\hline Punzón - Op 32 & Movimiento radial hacia la rama. & $\begin{array}{l}\text { Esfuerzos combinados sobre el pedúnculo al } \\
\text { actuador entrar en contacto con el fruto. }\end{array}$ & Actuador con superficie de punzones. \\
\hline Balancín - Op 46 & Movimiento axial hacia la rama. & Esfuerzos en la llegada del actuador. \\
\hline
\end{tabular}

Tabla 8. Características de las tres opciones priorizadas

Cada opción luego de ser diseñada y construida fue evaluada bajo los siguientes criterios:

Parámetros físicos de los frutos y el sistema fruto pedúnculo: características de los frutos que influyen en el funcionamiento del actuador:

- El estado de madurez de los frutos que rodean al fruto a desprender.

- Fuerza de unión entre frutos de un mismo glomérulo.

- Orientación del fruto respecto a la rama.

Parámetros de funcionamiento de los actuadores: características mecánicas y cinemáticas, de los actuadores explorados:
- Trayectoria seguida por la partes del mecanismo que se acercan al fruto.

- Linealidad de los ejes del fruto y del actuador.

- Partes del actuador que entran en contacto con el fruto maduro a cosechar.

- Desplazamiento de los frutos vecinos.

- Facilidad de sujeción del fruto seleccionado

- Duración del desprendimiento.

- Trayectoria del fruto desprendido.

\section{A. Actuador simple por torsión:}

Para generar torsión sobre cada fruto, se diseñó un sistema que consta de un cono invertido, y un micro-motor DC, que se encuentra girando constantemente, y al instante de tocar el fruto genera rotación del mismo y torsión en las fibras del sistema fruto pedúnculo, hasta finalmente desprender fruto. Figura 3.
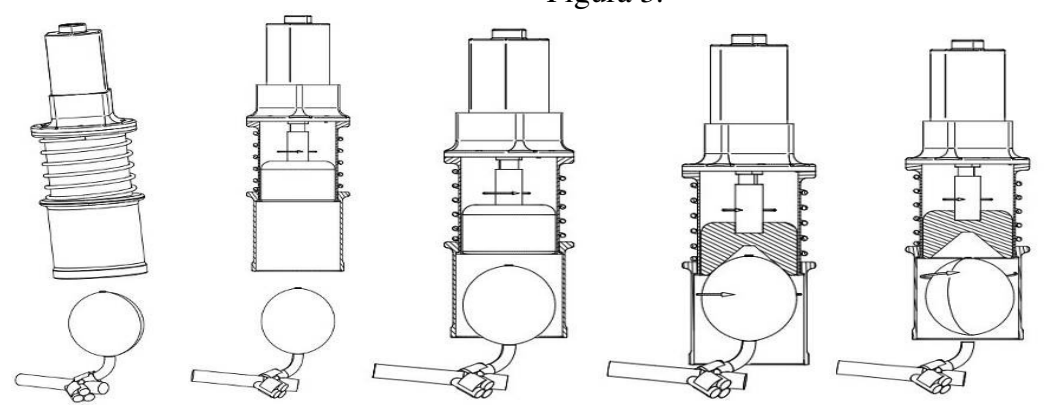

Figura 3. Secuencia de funcionamiento del actuador simple por torsión. Cono invertido. 
1) Construcción y exploración del actuador simple por torsión:

Este actuador de desprendimiento consta de dos sistemas generadores de movimiento independientes: el sistema de acercamiento o aproximación al fruto (Actuador lineal) y el sistema de generación de torsión (Micromotor eléctrico de alta potencia), Figura 4. Las dimensiones de las partes de este actuador fueron estimadas con relación al tamaño de los frutos de café Variedad Colombia. Se realizó la construcción y ensamble de 4 actuadores, Figura 4. Dentro del banco de pruebas de la Figura 4 se dispuso una rama de café sujeta por los extremos. El bastidor cilíndrico fue ubicado alineando los frutos cosechables de cada glomérulo con los actuadores. El desplazamiento del actuador lineal fue de $22 \mathrm{~mm}$ de forma radial a la rama, su posición inicial totalmente retraído está a los $5,2 \mathrm{~cm}$ del tallo de la rama y su posición final a $\operatorname{los} 3 \mathrm{~cm}$ del tallo de la rama.
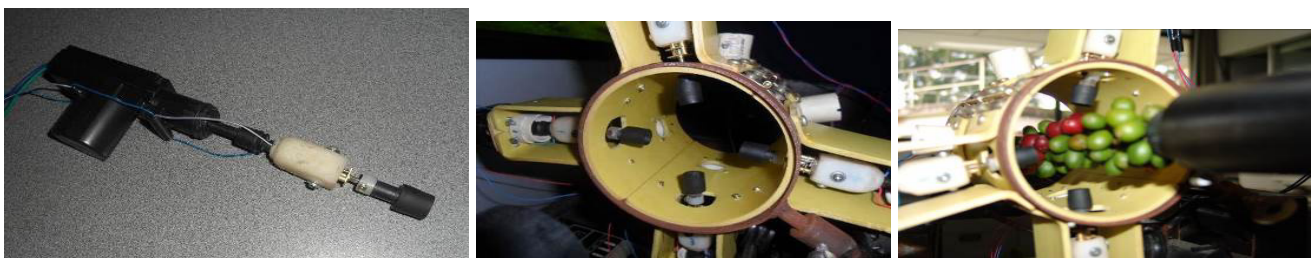

Figura 4. Herramienta Generadora de Torsión.

\section{B. Actuador con punzones de espetación:}

Esta opción consiste en un sistema tipo anzuelo donde cada actuador se introduce en cada fruto a cosechar por medio de un juego de punzones y al momento de retraer el actuador este desprende el fruto que ha sido espetado. Ver Figura 5.

\section{1) Construcción y exploración del actuador con punzones de espetación:}
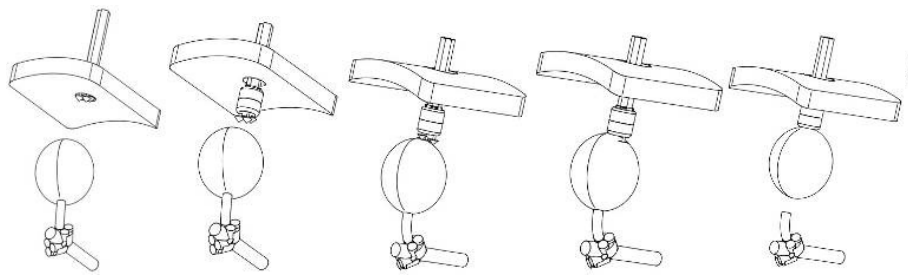

Figura 5. Secuencia de funcionamiento del actuador con punzones de espetación
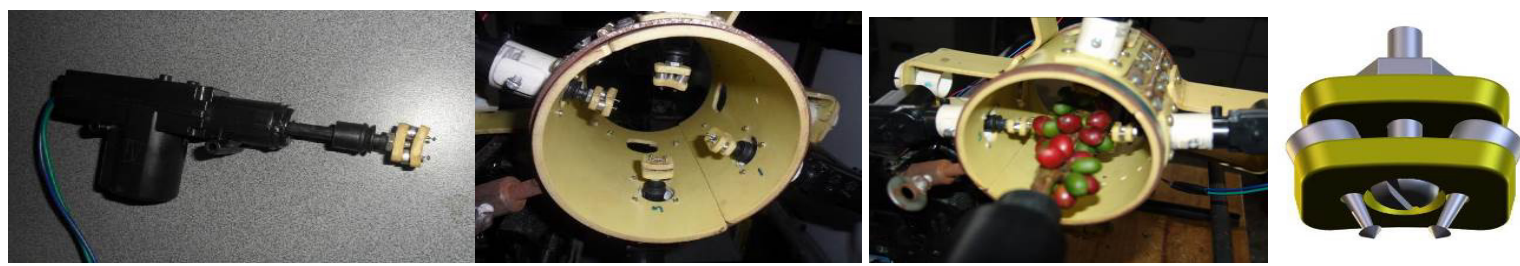

Figura 6. Herramienta Generadora de Tracción (Punzones de Espetación)

\section{Actuador tipo balancín:}

El actuador, con forma de paleta con punzones incrustados, se desplaza en un eje paralelo a la rama. En el momento que el fruto maduro sea identificado, el actuador se acerca a la rama incrustando sus punzones sobre el fruto, el cual es desprendido por efecto del desplazamiento relativo entre la
El actuador generador de tracción (Espetación), consta de un sistema generador de movimiento lineal alimentado con una batería de 12 voltios DC, Figura 6. En [6] Martínez et al. (2008) construyeron en el taller de Ingeniería Agrícola de Cenicafé punzones con diámetro máximo de $2 \mathrm{~mm}$, los cuales fueron incrustados y sujetados usando secciones de PVC de forma rectangular como se ve en la Figura 6, luego utilizando termoformado se proporcionó la forma cóncava buscando orientar las puntas de los punzones de tal manera que su proyección converja a un punto en el fruto de café a cosechar.

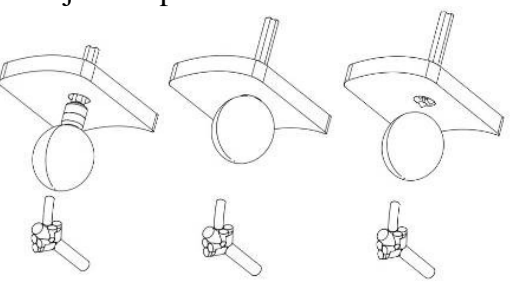

rama y la herramienta. En la Figura 7 se muestra un actuador escondido en la periferia de la herramienta y al momento de activarse se aproxima de forma axial y angular al eje de la rama. Este movimiento permite que el actuador ataque a uno de los costados superiores del fruto haciendo que el pedúnculo falle por esfuerzos combinados de cortante y momento flector. 


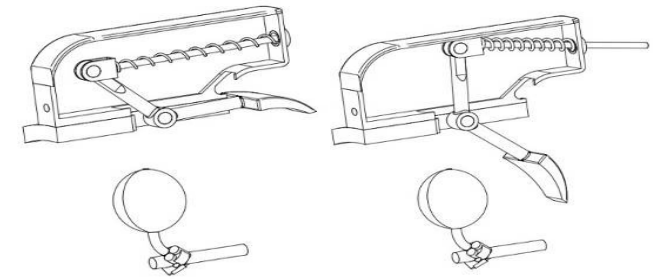

Figura 7. Secuencia de funcionamiento del actuador tipo balancín

1) Construcción y exploración del actuador tipo balancín:

El actuador generador de flexión, consta de un sistema generador de movimiento lineal alimentado con una batería de 12 VDC, Figura 8. Las dimensiones de las partes de este actuador fueron estimadas con relación al tamaño de los frutos
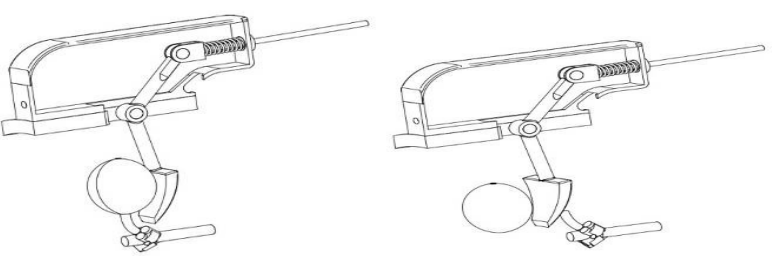

de café Variedad Colombia. La característica principal de este actuador es la forma cóncava del órgano y es posible que solo un fruto se encuentre en la zona de influencia de la concavidad para ser flectado y posteriormente desprendido.
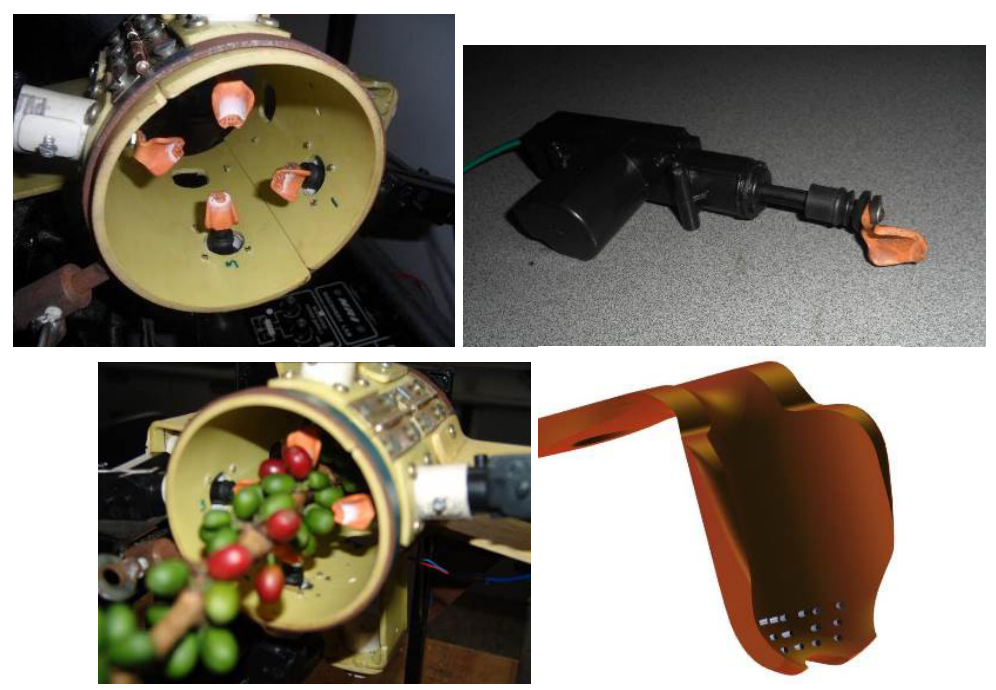

Figura 8. Herramienta Generadora de Flexión

\section{Evaluación de las opciones:}

Debido a que no se contaba con una opción patrón no se utilizó la matriz de PUGH para este caso y se realizaron pruebas de laboratorio para definir a partir del funcionamiento de cada opción cual era viable para cosechar frutos de forma individual. Cada sistema fue evaluado desprendiendo frutos de café en condiciones de laboratorio. En promedio cada sistema actuó sobre 33 frutos maduros con 17 frutos verdes que se encontraban en las vecindades, Tabla 9.

\begin{tabular}{cccc}
\hline Indicador & Torsión & Punzones & Balancín \\
Porcentaje de frutos & $60 \%$ & $0 \%$ & $50 \%$ \\
$\begin{array}{c}\text { maduros desprendidos } \\
\text { Porcentaje de frutos } \\
\text { verdes desprendidos }\end{array}$ & $20 \%$ & $0 \%$ & $43 \%$ \\
$\begin{array}{c}\text { Porcentaje de frutos } \\
\text { maduros no desprendidos } \\
\text { Porcentaje de frutos } \\
\text { verdes no desprendidos }\end{array}$ & $40 \%$ & $100 \%$ & $50 \%$ \\
\hline
\end{tabular}

Tabla 9. Desempeño de las opciones evaluadas.

Algunas consideraciones de los ensayos realizados son:
1. El sistema de espetación no es eficaz, no logra realizar ningún tipo de desprendimiento.

2. Los generadores de flexión y torsión son sistemas que muestran potencial, sin embargo, el desprendimiento de algunos frutos no fue total, pues parte del epicarpio del fruto maduro desprendido continua sosteniendo el fruto.

3. El sistema de torsión obtuvo el mejor desempeño de las tres opciones pues logró cosechar el mayor porcentaje de frutos maduros con la mínima incidencia de frutos verdes.

\section{E. Diseño detallado de la opción seleccionada:}

El sistema de desprendimiento individual de frutos debe acercarse al fruto maduros desde la periferia de la herramienta y a su vez lograr el desprendimiento del mismo.

\section{1) Optimización del sistema de desprendimiento:}

Para el desprendimiento el sistema de cono invertido, activado por un minimotor DC optimizado, Figura 9. 


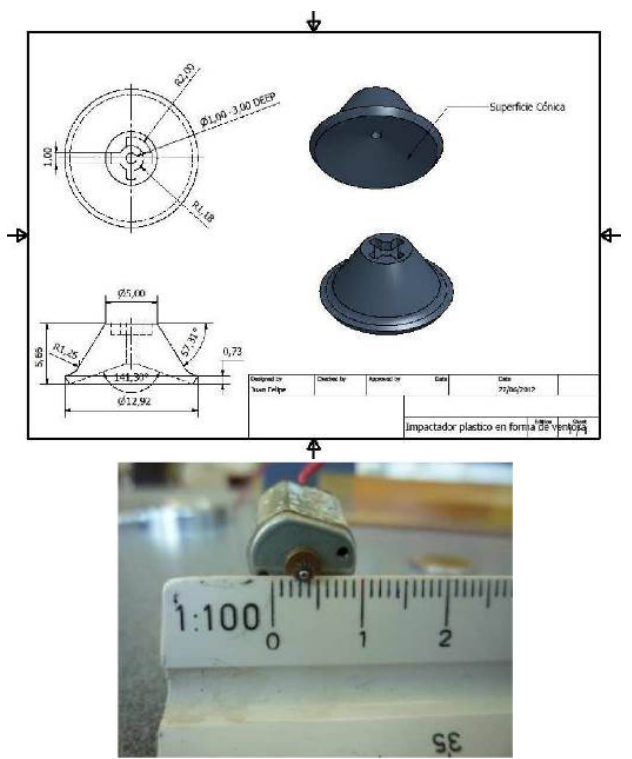

Figura 9. Cono invertido mecanizado y minimotor utilizado en el montaje.

\section{2) Diseño del sistema de acercamiento:}

Para la realización del sistema de acercamiento, el cual hace parte del sistema de desprendimiento, se analizó la lista de requerimientos, las cuales son: movimiento rápido y cíclico, compacto, de fácil construcción y mantenimiento, momentos de inercia mínimos y fácil de repetir a altas frecuencias de operación. Tomando en cuenta estas consideraciones de diseño se generaron tres opciones para el sistema: el mecanismo biela manivela corredera, una columna curva deslizante-oscilante y un cilindro telescópico, como se muestra en la Figura 10.

Se establece una ecuación del mecanismo biela-manivelacorredera para determinar las aceleraciones y velocidades del punto de interés, buscando que su componente radial en posición y velocidad, sean las mayores posibles en la zona de impacto. Para ello se sigue el esquema del mecanismo dado en la Figura 11. Se realiza la deducción de las ecuaciones que definen la trayectoria del punto $\mathrm{P}$ respecto a el movimiento de entrada Xo.

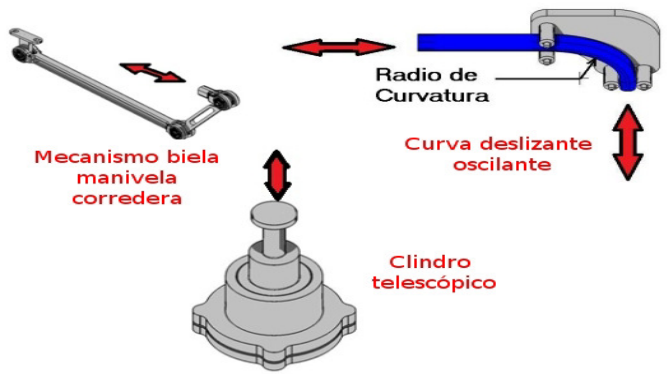

Figura 10. Mecanismos de acercamiento.

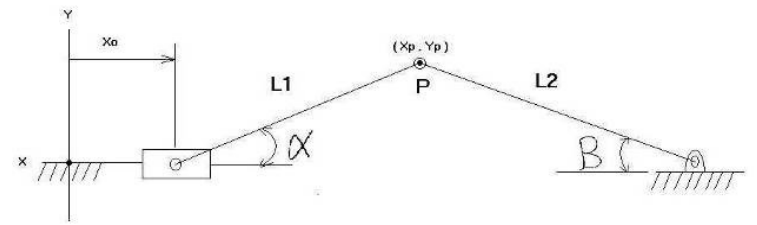

Figura 11. Esquema del Mecanismo de cuatro Barras para la deducción de la Ecuación 1.

Finalmente se determina que: conociendo las longitudes de los eslabones (constantes) y el punto inicial del deslizador (valor de entrada), quedan definidas las coordenadas del micromotor (salidas) y con las derivadas de la siguiente ecuación se puede conocer la velocidad y la aceleración del punto $\mathrm{P}$.

$$
\sin ^{-1}\left(\frac{L 2}{L 1} \sin (\beta)\right)=\cos ^{-1}\left(1-\frac{X o-L 2(1-\cos (\beta))}{L 1}\right)
$$

Ecuación 1

Siendo:

$$
\begin{aligned}
& X p=X o+L 1 \cos (\alpha) \\
& Y p=L 1 \sin (\alpha)=L 2 \sin (\beta) \\
& \alpha \text { y } \beta: \text { Ángulos que forman los eslabones con la } \\
& \text { horizontal. } \\
& X p \text { y } Y p: \text { Coordenadas (x,y) del micromotor } \\
& L 1 \text { y } L 2: \text { Longitudes de los eslabones } \\
& X o: \text { Posición inicial del deslizador. }
\end{aligned}
$$

En el punto $\mathrm{P}$ sobre uno de los eslabones, se ubica el micromotor. Para estudiar la trayectoria del mecanismo bielamanivela como sistema de acercamiento se realizaron algunos cálculos y síntesis gráfica usando software mecánico y geométrico (AutoCad Mechanical y Mechanical Desktop).

\section{3) Desarrollo de accionamientos para los sistemas de aproximación:}

Con el fin de proporcionar un movimiento de entrada para el mecanismo de cuatro barras se trabajó en la selección de un cilindro neumático y la construcción de un solenoide. También en la fabricación del cilindro neumático telescópico que logra reducir considerablemente el tamaño de la herramienta, que simplifica el acercamiento radial al eje de la rama si se compara con el mecanismo biela-manivela.

De acuerdo con los requerimientos de fuerza dados para el desprendimiento de frutos maduros, se diseñó un solenoide con un total de 3000 vueltas de alambre de cobre esmaltado 24AWG sobre un tubo plástico de $1 \mathrm{~cm}$ de diámetro y $9 \mathrm{~cm}$ de longitud, completando una resistencia eléctrica total de 22,96 Ohm y operando con una corriente de 0,57 A, en teoría, produce aproximadamente una fuerza electromotriz de $10,5 \mathrm{~N}$. Adicionalmente, se seleccionó dos cilindros neumáticos, uno de $100 \mathrm{~mm}$ de longitud, uno con diámetro de $8 \mathrm{~mm}$ y el otro de $10 \mathrm{~mm}$. Todos estos elementos descritos anteriormente pueden ser observados en la Figura 12. 


\section{4) Construcción y pruebas:}

Se construyó un banco de pruebas, el cual puede ser observado en Figura 13, el banco dispone de un montaje fabricado en tubo de PVC de cuatro pulgadas al cual se encuentra adherido el mecanismo de cuatro barras accionado por dos cilindros neumáticos y el solenoide desarrollado.

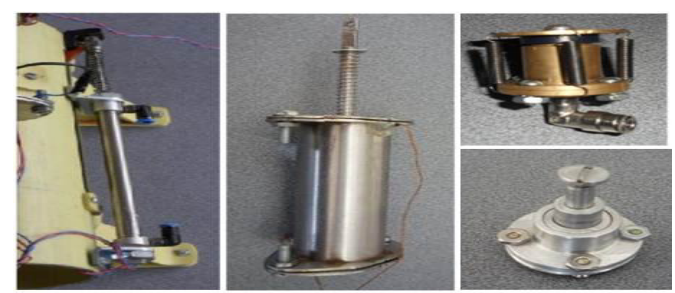

Figura 12. Sistemas de acercamiento cilindro neumático, solenoide y cilindro telescópico.

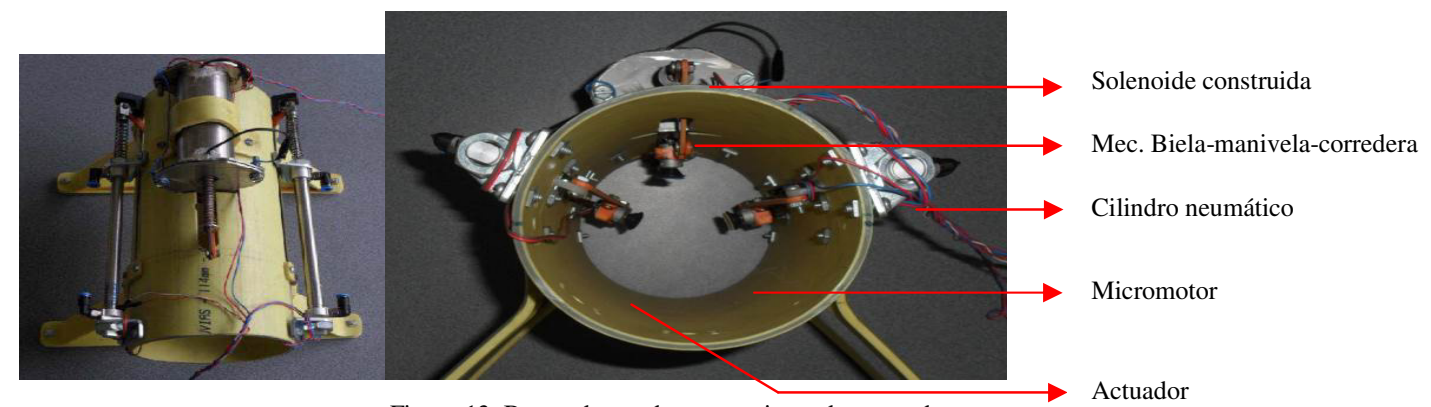

Figura 13. Banco de pruebas mecanismo de cuatro barras.

Los frutos en los glomérulos no siempre muestran un crecimiento en la dirección radia a la ramal, también pueden estar dispuestos en diferentes direcciones.

En estas pruebas, se estudió el ángulo de ataque que más desprendimientos causara, usando impactadores cónicos y con materiales de alto agarre como: látex de guantes quirúrgicos, látex corrugado y con incrustaciones de filamentos metálicos. En la Figura 14 se puede observar las configuraciones utilizadas.
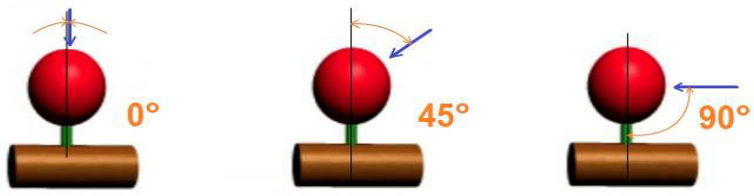

Figura 14. Ángulos de ataque impactador cónico torsional

El funcionamiento de cada ángulo de acercamiento y cada material fue probado sobre 10 frutos de café. La Tabla 10 muestra los resultados obtenidos, sobre los cuales el material con mayor potencial, fue el recubrimiento con látex quirúrgico.

\begin{tabular}{clc}
\hline Material & Ángulo & $\begin{array}{c}\text { Porcentaje total de } \\
\text { frutos maduros } \\
\text { desprendidos (\%) }\end{array}$ \\
\hline Filamentos metálicos & $0^{\circ}$ & 30 \\
incrustados. & $45^{\circ}$ & 10 \\
Recubrimiento látex & $90^{\circ}$ & 0 \\
quirúrgico. & $0^{\circ}$ & 50 \\
Recubrimiento látex & $45^{\circ}$ & 20 \\
corrugado. & $9^{\circ}$ & 10 \\
& $45^{\circ}$ & 30 \\
& $90^{\circ}$ & 0 \\
\hline
\end{tabular}

Tabla 10. Resultados de desprendimientos con el cono invertido y diferentes ángulos de llegada sobre el fruto

\section{CONSIDERACIONES Y CONCLUSIONES}

La metodología estructurada de diseño es una herramienta para el desarrollo de sistemas confiables y demuestra a partir de su aplicación el cumplimiento de las especificaciones del producto deseado, encontrando puntos críticos de ajuste, rediseño y reconceptualización del producto de ser necesario. Para el caso de procesos biológicos, considerados procesos estocásticos, la aplicación de dicha metodología tiene algunas complicaciones, pues el crecimiento, la disposición y la maduración de los frutos de café en una rama son muy variables y dependen en gran medida del clima, suelo y tipo de cultivo. Sin embargo, a partir de la selección y exploración de diferentes opciones para ser evaluadas en laboratorio, fue posible seleccionar la mejor opción.

El estado final de la opción diseñada de manera detallada, muestra en general un porcentaje de desprendimiento de frutos maduros del $60 \%$ y de frutos verdes del $20 \%$, en este último caso los desprendimientos fueron generados de manera accidental sobre frutos inmaduros que se encontraban en las vecindades de los frutos maduros alcanzados por el sistema.

Las pruebas realizadas al sistema de torsión evidencian que es necesario un ajuste a la opción seleccionada, con el fin de alcanzar un desprendimiento de frutos maduros superior al $90 \%$.

Las observaciones realizadas en los experimentos muestran que, las características físicas, geométricas y mecánicas del sistema fruto-pedúnculo y del glomérulo se deben considerar para el diseño de una herramienta, que permita el desprendimiento selectivo de los frutos maduros. El sistema fruto-pedúnculo se caracteriza por tener alta flexibilidad, 
resiliencia $^{1}$ y tenacidad ${ }^{2}$, cuando una carga es aplicada directamente sobre un fruto de café, el pedúnculo se deformará haciendo que éste se desvíe permaneciendo unido al pedúnculo sin que se produzca el desprendimiento. A esta situación se adiciona el hecho que la orientación del eje ecuatorial de un fruto no siempre es radial a la rama. Otro aspecto a tener en cuenta es la condición de frontera que generan los frutos vecinos al fruto maduro objetivo, el actuador puede aplicar la carga sobre este último, sin embargo puede también hacerlo sobre los vecinos y accidentalmente generar desprendimientos de frutos verdes.

La activación de la opción desarrollada para cosecha de café se realizó de forma manual, por esto se plantea como recomendación, desarrollar un sistema de control que guíe automáticamente los actuadores hacia los frutos de café maduros, de esta manera el porcentaje de frutos maduros desprendidos podría aumentar y el porcentaje de frutos inmaduros podría disminuir.

Se requiere ampliar el estudio del comportamiento de los frutos al ser expuestos a esfuerzos por tracción, con el fin de lograr un desprendimiento eficiente y eficaz. Dicho estudio podría requerir una alta inversión de tiempo, por lo que dentro del proyecto se plantea utilizar la experiencia adquirida en el desarrollo de equipos de cosecha semimecanizada para café, rediseñarlos para la aplicación de cosecha selectiva y lograr en corto tiempo una herramienta que sea evaluada en campo.

La situación anterior, indica que por ahora, un sistema de desprendimiento individual, por si solo, no es una opción viable técnica y económicamente para cosechar café de forma selectiva; sin embargo se muestra un proceso de creatividad e innovación que con ajustes y rediseños podría mas adelante tener un alto potencial de ser aplicado.

\section{AGRADECIMIENTOS}

Los autores expresan sus agradecimientos al Departamento Nacional de Ciencia, Tecnología e Innovación - Colciencias, por la cofinanciación del proyecto titulado "Desarrollo de una herramienta portátil con visión artificial para la cosecha selectiva de café", sobre el cual se desarrollaron las actividades descritas en este artículo. De igual forma agradece a la Universidad Tecnológica de Pereira y a la Universidad Autónoma de Manizales por la participación en este proceso a través del desarrollo de prácticas universitarias conducentes a trabajos de grado con dos de sus estudiantes.

\footnotetext{
${ }^{1}$ Resiliencia: Capacidad de un material de recibir energía antes de deformase permanentemente.

${ }^{2}$ Tenacidad Capacidad de un material de recibir energía antes de romperse.
}

\section{REFERENCIAS}

[1] ARISTIZABAL T., I.D.; OLIVEROS T., C.E.; ALVAREZ M., F. Propiedades físico-mecánicas del árbol de café y su relación con la mecanización de la cosecha. Cenicafé (Colombia) 50(4):313-326. 1999.

[2] CARDONA D., J.A. Diseño de una máquina portátil para la cosecha asistida de café. Pereira (Colombia), Universidad Tecnológica de Pereira. Facultad de Ingeniería Mecánica, 2006. 147 p. Tesis: Ingeniero Mecánico.

[3] FRONCZAK, F.J. Product Design. ME-549 Lecture Notes. Department of Mechanical Engineering of Wisconsin - Madison. Madison, WI. 1999.

[4] LONDOÑO H., D.; OLIVEROS T., C.E.; MORENO S., M.A. Desarrollo de una herramienta manual para asistir la recolección de café en Colombia. Cenicafé (Colombia) 53(2):93-105. 2002.

[5] LOPEZ F., H.A.;ROA M., G.;PARRA C., A.; Evaluación del equipo "Aroandes", un prototipo para la cosecha manual asistida de café. Revista Cenicafé, 2006, pp 208-219.

[6] MARTÍNEZ C., V.M.; SANZ U., J. R; OLIVEROS T., C. E.; MORENO C., E. L. Respuesta de los frutos de café a la espetación. Cenicafé 59(1):64-74.2008.

[7] OLIVEROS T., C.E; RAMIREZ G., C.A; ACOSTA. A., R.; ALVAREZ M., F. Equipo portátil para asistir la cosecha manual de cosecha de café. Revista Facultad Nacional de Agronomía, Medellín, Vol . 58, No 2, 2005, pp 3003-3013.

[8] PUGH, S. (1991). Total design: Integrated methods for successful product engineering. Wokingham, England: Addison-Wesley Pub. Co.

[9] QUIÑÓNEZ O.,W. Evaluación del equipo ALFA, In: CENTRO NACIONAL DE INVESTIGACIONES DEL CAFÉ-Cenicafé. Chinchiná, Colombia, Informe anual de actividades, Disciplina de Ingeniería agrícola. 2010, Chinchiná, Cenicafé, 2010.

[10] RAMIREZ G., C.A.; OLIVEROS T., C.E; SANZ U., J.R; ACOSTA. A., R.; BUENAVENTURA A. J.D. Desgranador mecánico para la cosecha de café. DESCAFÉ. Revista Cenicafé, 2006, pp 121-133.

[11] SALAZAR A., J.N.; OROZCO C., F.J.; CLAVIJO P., J.F. Características morfológicas, productivas y componentes del rendimiento de dos variedades de café: Colombia y Caturra. Cenicafé (Colombia) 39(2):43-60. 1988.

[12] SANZ U., J.R. Development of a mobile device for coffee harvesting suitable for the colombian conditions. Madison (Estados Unidos), University of Wisconsin. Department of Mechanical Engineering, 2003. 175 p. Tesis: PhD. 